\title{
FOLFIRINOX regulated tumor immune microenvironment to extend the survival of patients with resectable pancreatic ductal adenocarcinoma
}

\author{
Meng-Yao Wu, Meng Shen, Meng-Dan Xu, Zheng-Yuan Yu, Min Tao \\ Department of Oncology, First Affiliated Hospital of Soochow University, Suzhou, China \\ Contributions: (I) Conception and design: M Tao, MY Wu; (II) Administrative support: ZY Yu; (III) Provision of study materials or patients: MD \\ $\mathrm{Xu}$; (IV) Collection and assembly of data: M Shen; (V) Data analysis and interpretation: MY Wu; (VI) Manuscript writing: All authors; (VII) Final \\ approval of manuscript: All authors. \\ Correspondence to: Dr. Min Tao; Dr. Zheng-Yuan Yu. Department of Oncology, First Affiliated Hospital of Soochow University, 899 Pinghai Road, \\ Suzhou, China. Email: mtao@medmail.com.cn; strongeryy1985@163.com.
}

Background Pancreatic ductal adenocarcinoma (PDAC) is one of the most deadly malignant tumors
worldwide due to its ineffective diagnosis and poor prognosis. The longest median overall survival (OS)
to PDAC patients has been provided by FOLFIRINOX. It is essential to identify the mechanisms of
FOLFIRINOX to gain new insights for the treatment of PDAC.

Methods: We compared gene expression levels of PDAC patients who received neoadjuvant FOLFIRINOX prior to surgery with those of patients who received no neoadjuvant chemotherapy. Bioinformatics analysis was applied to screen differentially expressed genes (DEGs). Three microarray data sets were downloaded to analyze gene expression data between PDAC and adjacent non-tumor tissues. Overlapping DEGs were subjected to Kaplan-Meier survival analysis. The genes relating to poor outcomes and would be decreased after FOLFIRINOX were input into the Oncomine, University of Alabama Cancer (UALCAN), and LinkedOmics databases to analyze the gene expression and regulation networks.

Results: A total of 83 differentially expressed genes (DEGs) were screened and subjected to bioinformatics analysis, which indicated FOLFIRINOX influenced the immune microenvironment of PDAC. Seventythree genes significantly associated with the OS of PDAC patients. A Venn diagram revealed CXCL5 and PLAU were related to poor outcomes and would decrease after FOLFIRINOX chemotherapy of PDAC patients. It turned out that CXCL5 participated in the immune response-regulating signaling pathway in PDAC patients.

Conclusions: FOLFIRINOX regulated tumor immunity by reducing expression of the immunosuppressive gene CXCL5, laying a foundation for further study of combination therapy of FOLFIRINOX and immunotherapy.

Keywords: Pancreatic ductal adenocarcinoma (PDAC), FOLFIRINOX, immune microenvironment, bioinformatics analysis, overall survival (OS)

Submitted Oct 12, 2020. Accepted for publication Dec 09, 2020.

doi: 10.21037 /gs-20-828

View this article at: http://dx.doi.org/10.21037/gs-20-828

\section{Introduction}

Pancreatic cancer is an aggressive and deadly disease with poor clinical prognosis. Over $80 \%$ of patients suffering from pancreatic cancer have incurable disease at the time of diagnosis. The standard treatments for pancreatic ductal adenocarcinoma (PDAC) patients is the use of cytotoxic chemotherapy as well as surgical resection in the early stages (1). 
In phase I and phase II/III clinical trials, modified 5-fluorouracil, leucovorin, irinotecan, and oxaliplatin (FOLFIRINOX) has shown significant clinical benefits over gemcitabine alone (2). For patients with resectable disease, FOLFIRINOX is the standard-of-care adjuvant therapy, and it has been proven to exhibit the longest median overall survival (OS) (54 months) (3). Neoadjuvant treatment strategies have shown improved outcomes according to data from several randomized trials (1). However, the mechanism accounting for FORFIRINOX in pancreatic cancer treatment is not well-reported, and it needs to be evaluated in order to expand its application.

In this study, we used integrated bioinformatics methods to find the differentially expressed genes (DEGs) after FOLFIRINOX treatment of pancreatic cancer patients. By analyzing open access available bioinformatics resources, we found that FOLFIRINOX regulated tumor immunity. In addition, the key protein $\mathrm{C}-\mathrm{X}-\mathrm{C}$ motif chemokine 5 (CXCL5) was identified in our study, which established an immunosuppressive microenvironment in PDAC.

We present the following article in accordance with the MDAR reporting checklist (available at http://dx.doi. org/10.21037/gs-20-828).

\section{Methods}

\section{Microarray data and identification of DEGs}

A microarray data set (GSE129492) consisting of the gene expression data of patients who received upfront surgical resection (no neoadjuvant therapy, $n=6$ ) and patients who received neoadjuvant FOLFIRINOX $(n=6)$ was obtained from the Gene Expression Omnibus (GEO). The DEGs were identified by classical $t$-test with a false discovery rate of $<0.05$ and fold change (FC) $>1.5$ as the cutoff criterion. A TIGR Multi Experiment Viewer (The Institute for Genomic Research, Rockville, MD, USA) was applied by average linkage algorithm to perform samples hierarchical clustering. The GSE15471, GSE16515, and GSE28735 microarray data sets were downloaded to analyze gene expression data between PDAC and adjacent non-tumor tissues. The DEGs were identified by GEO2R with a false discovery rate of $<0.05$ and $\log F C>1$.

\section{Functional and patbway enrichment analysis, and PPI network construction}

The functional enrichment analysis tool FunRich (version 3.1.3 for Windows; http://www.funrich.org/) was used to perform Gene ontology (GO) enrichment analysis of the identified DEGs. All genes were uploaded to construct protein-protein interaction (PPI) in the hub module of the Search Tool for the Retrieval of Interacting Genes (STRING) database (http://string-db.org/), a popular software which was always used to produce integrated models of biomolecular interaction networks.

\section{OS analysis}

To analyze the prognostic value of the candidate genes, the PDAC participants were stratified into high and low expression groups for each DEG, using the median expression level from The Cancer Genome Atlas (TCGA, https://cancergenome.nih.gov/) as the cutoff. The two participant cohorts for each DEG were compared by Kaplan-Meier analysis and the log-rank test using the $\mathrm{R}$ survival package (https://CRAN. R-project. org/ package=survival).

\section{Genes efficacy evaluation}

The database Oncomine (https://www.oncomine.org/) was used to testify the transcriptional level of genes expression. This analysis utilized several PDAC study datasets, including the Badea Pancreas, Ishikawa Pancreas, Logsdon Pancreas, Pei Pancreas, Segara Pancreas, Grutzmann Pancreas, and Iacobuzio-Donahue Pancreas studies. Relative expression of genes based on clinicopathological features was analyzed on UALCAN (http://ualcan.path.uab. edu), an interactive portal which was applied to perform deep analysis of the TCGA database. The DEGs were studied to mine the genes related with the TCGA PDAC cohort using the Link Finder module of the LinkedOmics database (http://www.linkedomics.org/). We then performed GO (cellular component, biological process, and molecular function) and Kyoto Encyclopedia of Genes and Genomes (KEGG) pathways analyses of related genes by gene set enrichment analysis (GSEA).

\section{Ethic}

The study was conducted in accordance with the Declaration of Helsinki (as revised in 2013).

\section{Statistical analysis}

Survival analyses were performed using the method of 
Kaplan-Meier for the entire cohort. An unpaired Student's $t$-test was used for comparing levels of CXCL5 and PLAU between different subgroups. The correlations between different genes were analyzed using Spearman's rank test. A $P$ value $<0.05$ was considered to be statistically significant.

\section{Results}

\section{Neoadjuvant FOLFIRINOX was associated with immunologically relevant alterations within PDAC tumors}

A total of 83 DEGs including 26 upregulated and 57 downregulated genes were screened from GSE129492 containing 6 pancreatic cancer patients who had received neoadjuvant FOLFIRINOX prior to surgery, and 6 participants who received no treatment prior to surgery (Figure 1A). Functional enrichment analysis was performed to investigate the function of DEGs using FunRich software (version 3.1.3 for Windows; http://www.funrich.org/). The results demonstrated that the DEGs were mainly enriched in the following cellular components: plasma membrane, extracellular space, extracellular (Figure 1B); biological processes: immune response, signal transduction, cell communication (Figure 1C); and molecular functions: major histocompatibility complex (MHC) class I receptor activity, MHC class II receptor activity, T cell receptor (TCR) activity (Figure 1D). Subsequently, we verified a biological pathway enriching the DEGs in following: immune system, cytokine signaling in immune system, innate immune system, TCR signaling in CD8 ${ }^{+} \mathrm{T}$ cells, CD40/CD40L signaling, and TCR signaling in $\mathrm{CD}^{+}{ }^{+} \mathrm{T}$ cells (Figure $1 E$ ). The PPI network of DEGs was comprised of 83 nodes and 418 edges, the network had significantly more interactions than expected (Figure 1F).

\section{OS related genes CXCL5 and urokinase plasminogen activator decreased after chemotherapy of FOLFIRINOX}

A total of three microarray data sets of pancreatic cancer and adjacent non-tumor tissues (GSE16515, GSE15471, and GSE28735) were downloaded from the GEO database and analyzed. We found that GSE16515 from 36 PDAC and 16 adjacent non-tumor tissues exhibited 1,351 upregulated DEGs and 473 downregulated DEGs in PDAC compared with normal tissues. The GSE15471 dataset from $36 \mathrm{PDAC}$ and paired adjacent non-tumor tissues exhibited 1,790 DEGs, 1,557 with higher expression and 233 with lower expression. The GSE28735 dataset contained data from 45 PDAC and paired adjacent non-tumor tissues identified 412 DEGs, of which 256 were upregulated and 156 were downregulated (Figure $2 A$ ).

A Venn diagram was performed based on the 3 datasets, and 197 overlapping upregulated DEGs and 66 overlapping downregulated DEGs were identified (Figure 2B,C). Furthermore, overlapping DEGs were subjected to Kaplan-Meier survival analysis. Lower expression of 72 upregulated DEGs (Figure S1) was associated with longer survival, and only 1 downregulated DEG correlated with PDAC prognosis. A Venn diagram was applied to analyze overlapping genes (Figure 2D). There was upregulation of $C X C L 5$ and urokinase plasminogen activator (PLAU) in PDAC compared to normal tissue, and correlated with patient prognosis (Figure 2E,F). At the same time, both of them decreased after chemotherapy of FOLFIRINOX.

\section{CXCL5 participated in the immune response-regulating signaling pathway in PDAC patients}

Transcription levels of CXCL5 were detected in multiple PDAC studies from TCGA and GEO. The expression level of CXCL5 was significantly higher in pancreatic carcinoma compared to normal tissues $(\mathrm{P}<0.05)$ based on the Oncomine database (Figure $3 A$ ). Further subgroup analysis of multiple clinic pathological features indicated that the transcription level of CXCL5 in PDAC patients was significantly elevated compared to healthy people based on age, gender, race, tumor grade, disease stage, pancreatitis status, and tumor protein (TP) 53 mutation status (Figure 3B,C,D,E,F,G,H). Thus, CXCL5 expression might serve as a potential diagnostic indicator in PDAC.

Subsequently, we analyzed in-depth the mRNA sequencing data from PDAC patients in the TCGA to search related genes of $C X C L 5$ with the Function module of LinkedOmics (http://linkedomics.org/). The volcano plot (Figure 3I) indicated that 874 genes (dark red dots) showed significant positive correlations with CXCL5, whereas 730 genes (dark green dots) showed significant negative correlations [false discovery rate $(\mathrm{FDR})<0.01$ ]. Here, we exhibited the 50 most significant gene sets negatively and positively correlated with CXCL5 in the heat map (Figure 37,K). The DEG sets in correlation with CXCL5 were performed to GSEA. Significant GO term analysis revealed that genes relating to CXCL5 were located mainly in the specific granule, tertiary granule, and MHC protein complex, where they participate primarily in leukocyte cell- 
A

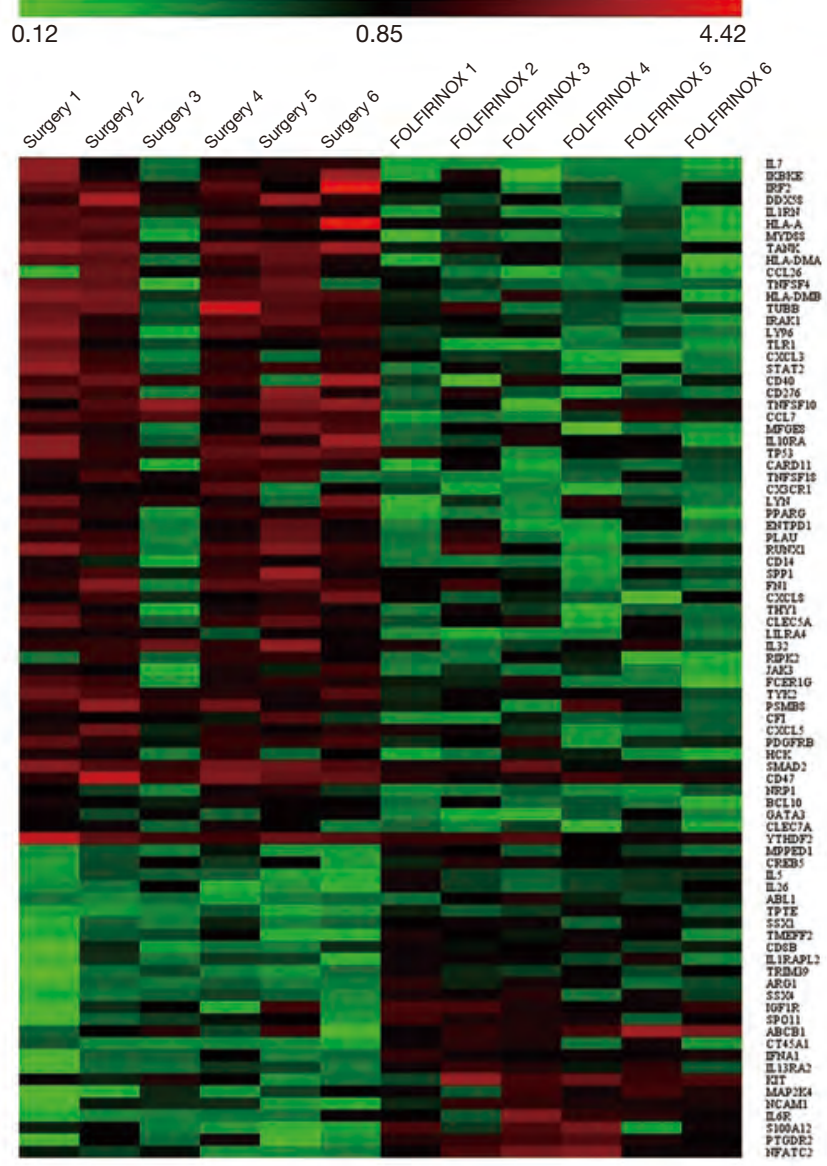

B

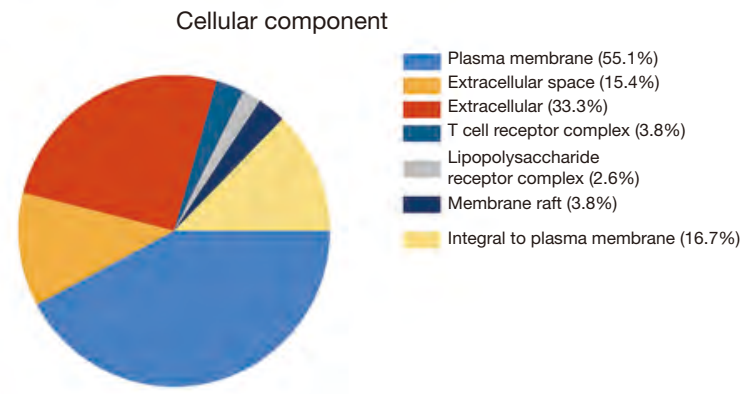

C

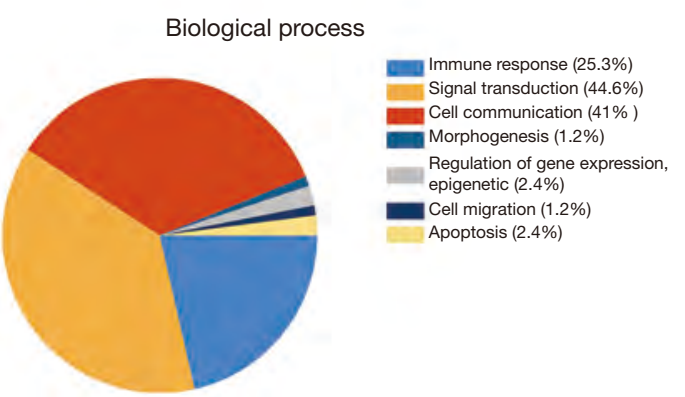

D

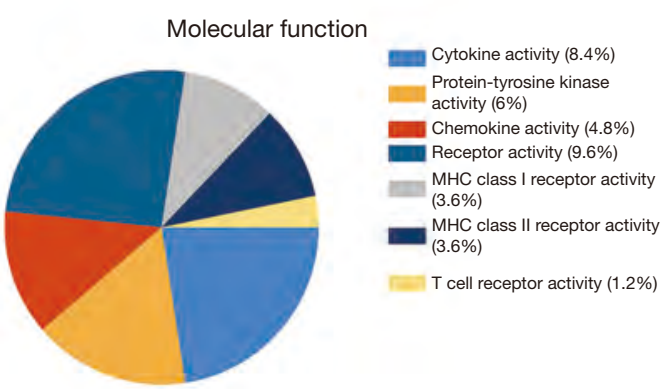

$\mathrm{F}$
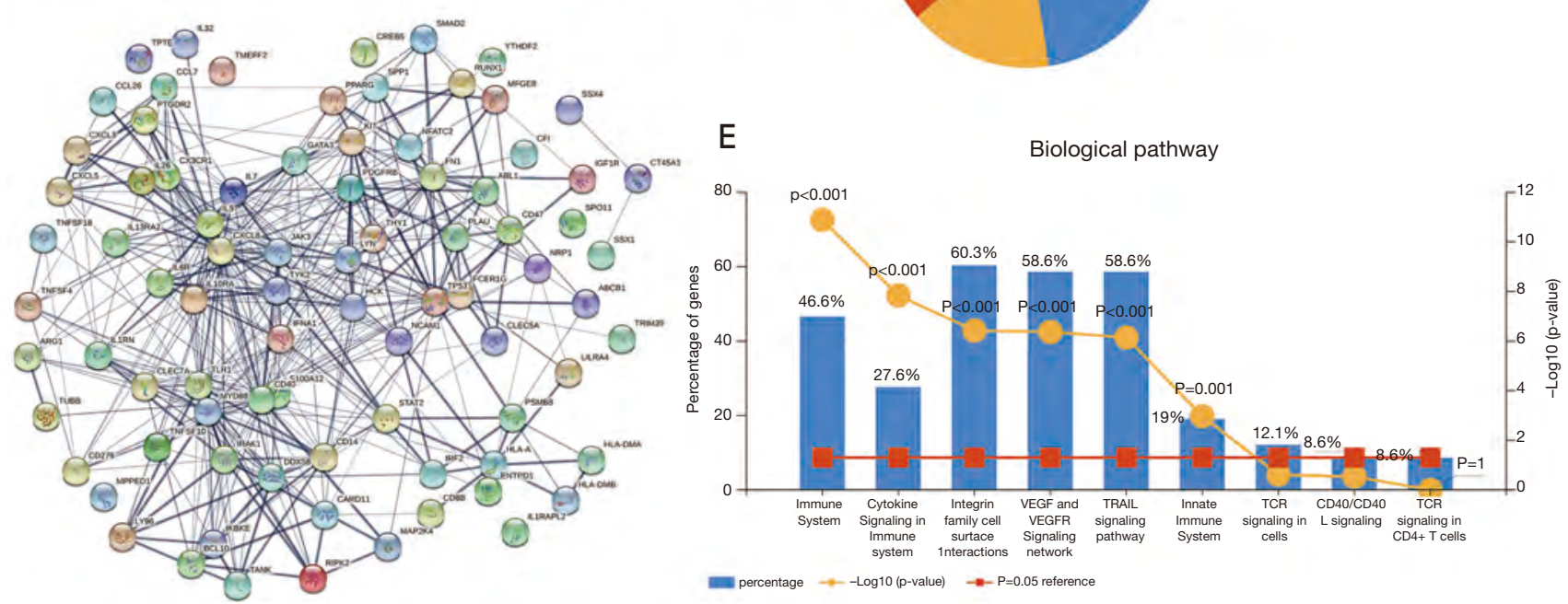

Figure 1 Neoadjuvant FOLFIRINOX was associated with immunologically relevant alterations within PDAC tumors. Heatmaps of 83 DEGs between 6 pancreatic cancer patients who received neoadjuvant FOLFIRINOX prior to surgery and 6 patients who received no treatment prior to surgery. (A) Red indicates upregulation and green indicates downregulation; (B) cellular component of identified DEGs was performed by FunRich; (C) biological process of identified DEGs; (D) molecular function of identified DEGs; (E) biological pathway of identified DEGs; (F) PPI network of the DEGs. PDAC, pancreatic ductal adenocarcinoma; DEGs, differentially expressed genes; PPI, protein-protein interaction. 

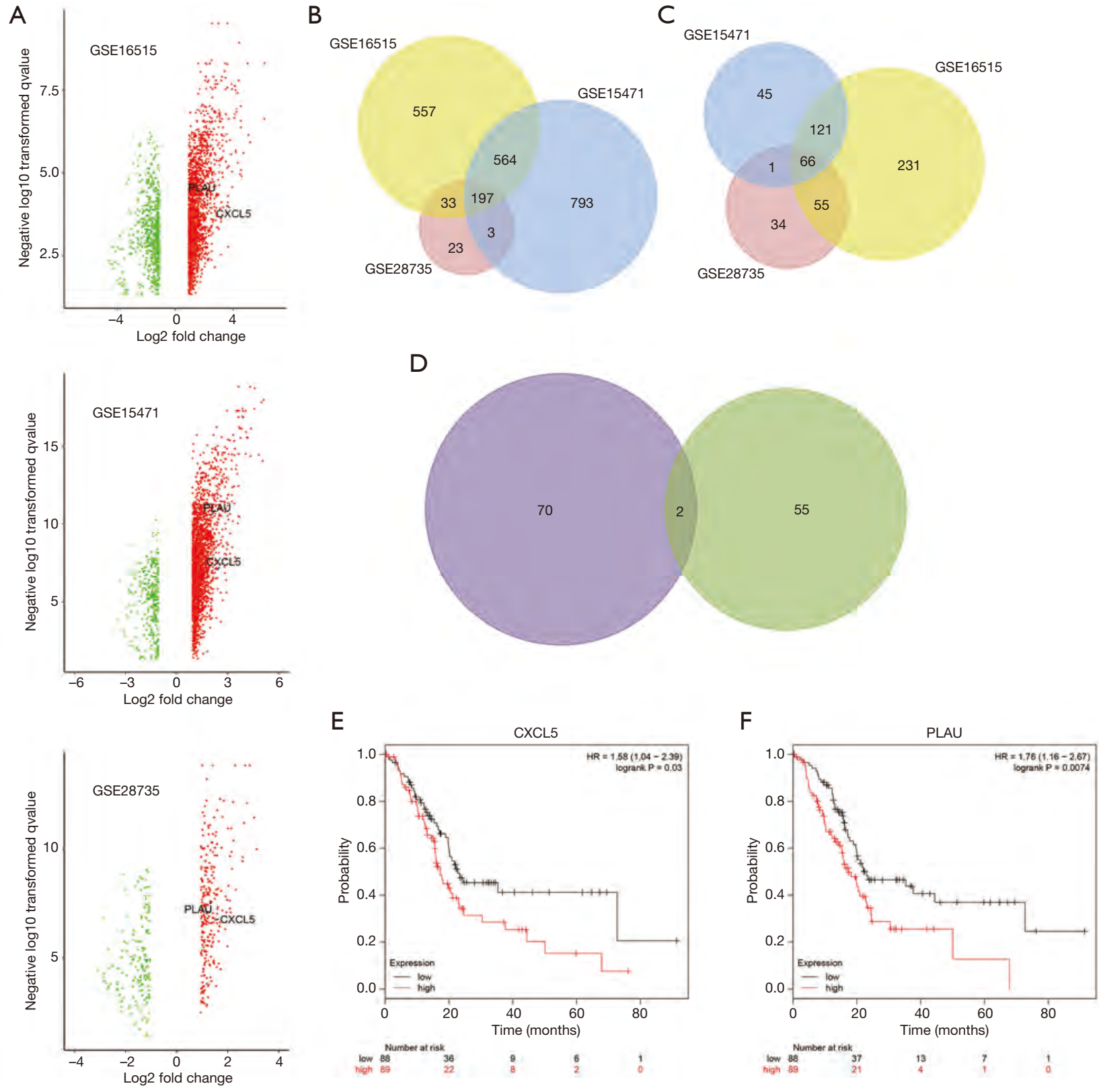

Figure 2 OS-related genes CXCL5 and PLAU decrease after chemotherapy of FORFIRINOX. (A) Identification of DEGs in GSE15471, GSE16515, and GSE28735. Red indicates upregulation and green indicates downregulation; (B) Venn diagram of 197 overlapping upregulated DEGs; (C) Venn diagram of 66 overlapping downregulated DEGs; (D) identification of DEGS upregulated in PDAC compared to normal tissue, correlated with patient prognosis and decreased after chemotherapy of FORFIRINOX; (E) Kaplan-Meier survival analysis of CXCL5; (F) Kaplan-Meier survival analysis of PLAU. OS, overall survival; DEGs, differentially expressed genes; PDAC, pancreatic ductal adenocarcinoma.

cell adhesion, neutrophil mediated immunity, and immune response-regulating signaling pathway. They were involved in the extracellular matrix (ECM) structural constituent, cytokine binding, and immunoglobulin binding. At last, enrichment genetic pathways were shown by KEGG pathway analysis to be involved in the staphylococcus aureus infection, osteoclast differentiation, and antigen processing and presentation (Figure $3 L$ ). 


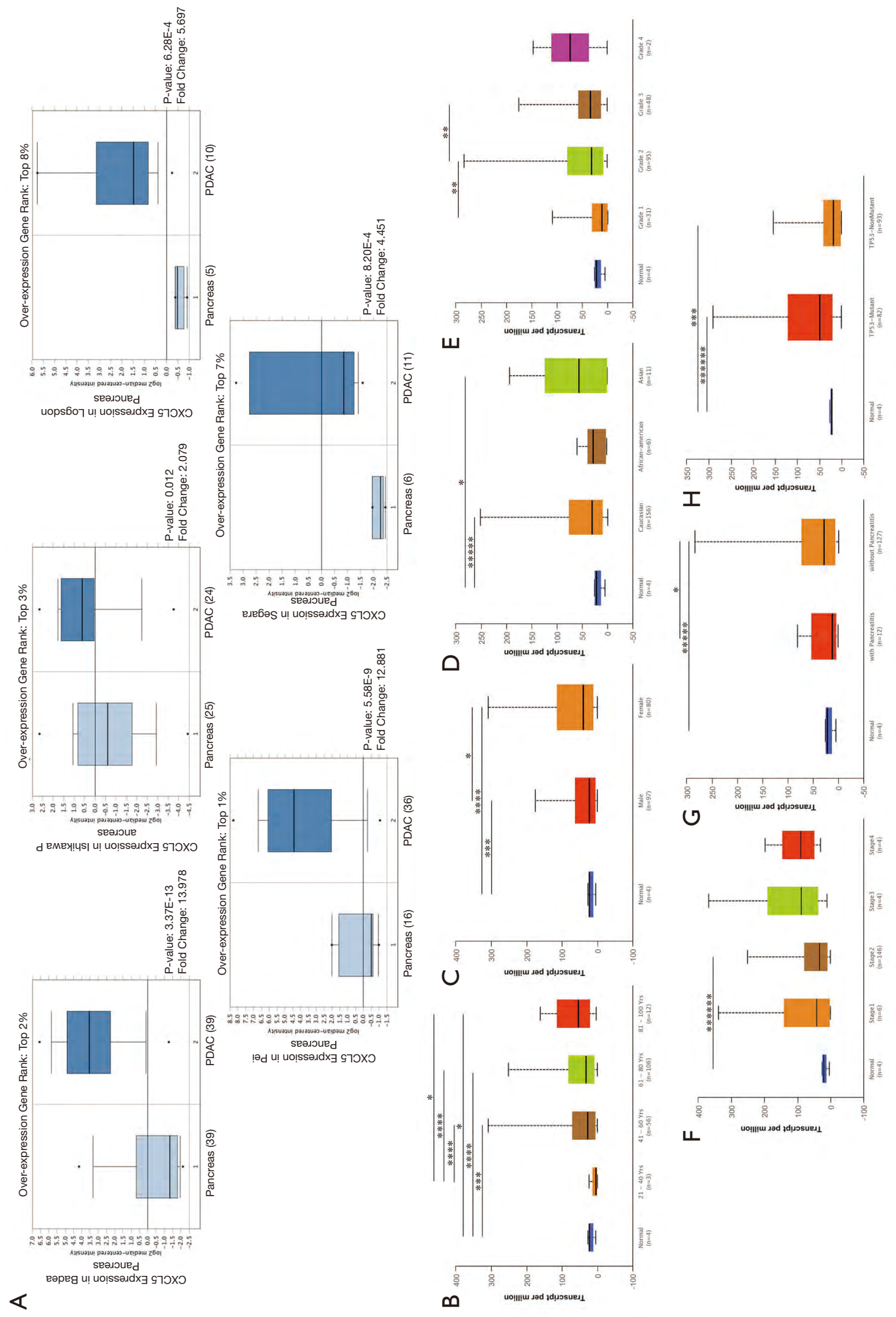



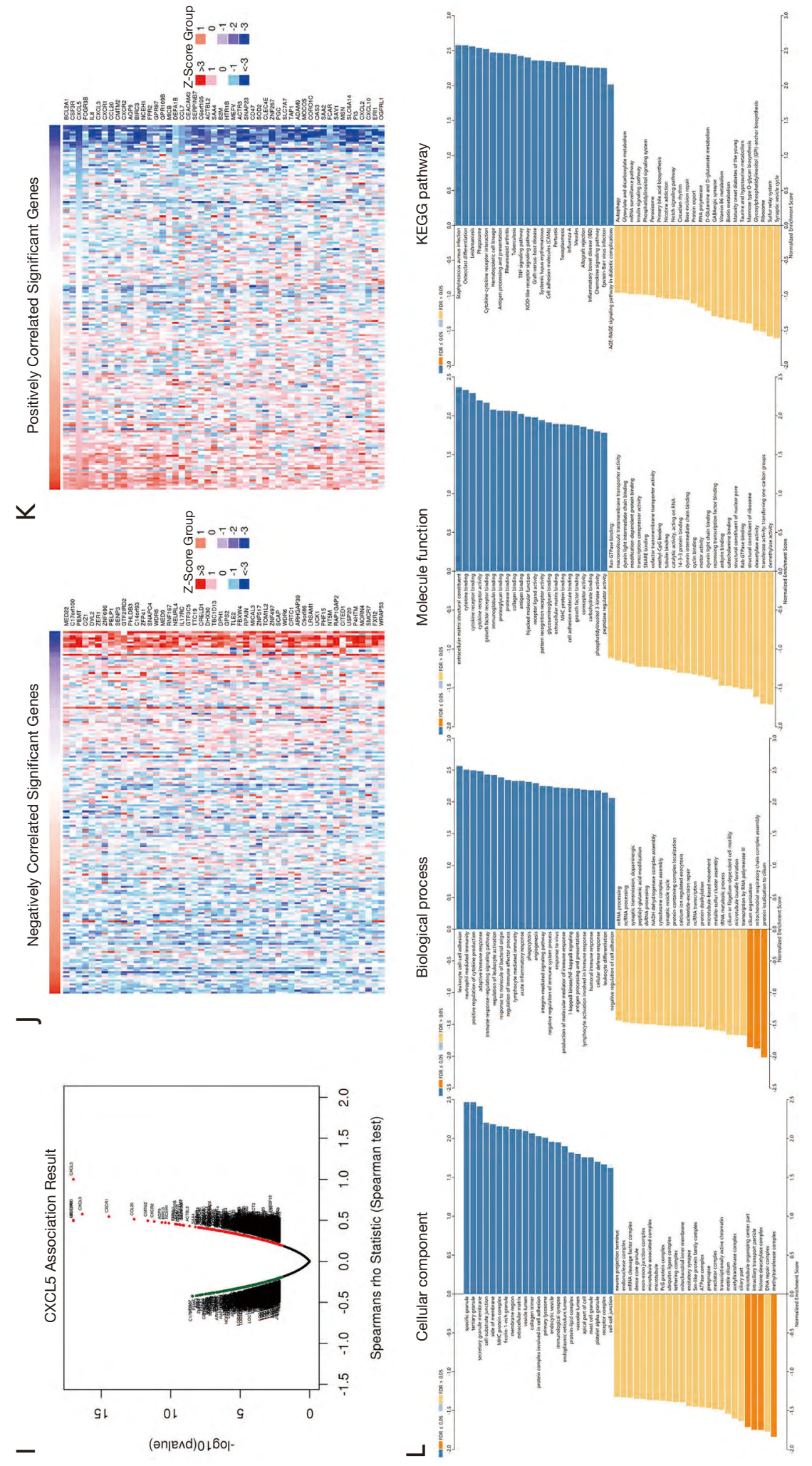

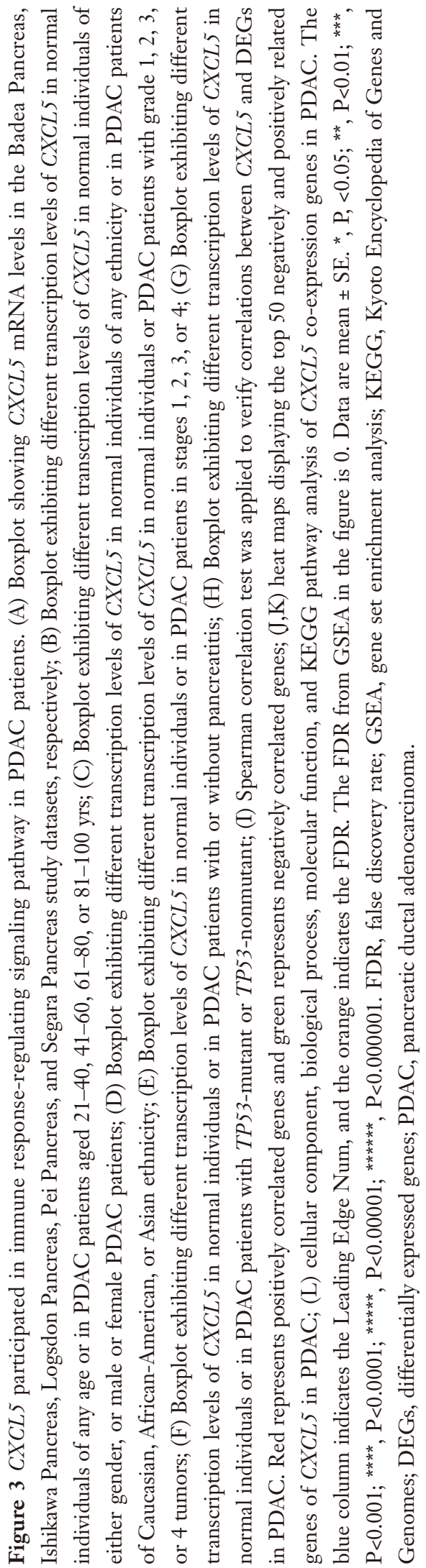




\section{PLAU expression and co-expression genes correlated with PLAU in PDAC}

Transcription levels analyses of PLAU were similar to CXCL5. The expression of PLAU was significantly increased in PDAC tissues compared to normal tissues $(\mathrm{P}<0.05)$ (Figure 4A). Subgroup analysis indicated that the PLAU transcription level of PDAC patients was significantly raised in comparison to healthy people based on tumor grade, disease stages, and TP53 mutation status (Figure 4B,C,D).

The volcano plot (Figure $4 E$ ) exhibited 2,805 genes (dark red dots) that were significantly positively related to $P L A U$, and 2,750 genes (dark green dots) significantly negatively related to PLAU (FDR <0.01). The 50 significant gene sets negatively and positively correlated with $P L A U$ are shown in the heat map (Figure $4 F, G$ ). The DEG sets correlated with PLAU were subjected to GSEA. The results showed that DEGs correlated with PLAU were located mainly in the ECM, collagen timer, and cell-substrate junction, where they participate primarily in extracellular structure organization, collagen metabolic processes, and bone development. The molecular functions of the genes were involved in ECM structural constituent, collagen binding, and cell adhesion molecular binding. The KEGG pathway analysis was mainly enriched in ECM-receptor interaction, focal adhesion, and protein digestion and absorption (Figure 4H).

\section{Discussion}

In this paper, datasets comparing gene expression levels of PDAC patients who received neoadjuvant FOLFIRINOX prior to surgery and patients who did not undergo neoadjuvant chemotherapy were firstly reviewed in the GEO database. The enrichment analysis of biological function and pathway was performed using GO and KEGG. According to the results, FOLFIRINOX could affect 83 DEGs, and influence the immune microenvironment of PDAC. We further sought to discover key genes among these 83 DEGs which were related to the progression of PDAC and associated with the outcome of PDAC patients. Therefore a holistic search of the GEO database was performed to acquire DEGs between pancreatic cancer and adjacent non-tumor tissues using GEO2R. A total of 263 overlapping genes were obtained and subjected to Kaplan-Meier survival analysis relying on gene expression in the TCGA PDAC database. As a result, 73 genes were significantly associated with the OS of PDAC patients.
Ultimately, the comparisons revealed CXCL5 and PLAU were related to poor outcomes and would be decreased after FOLFIRINOX chemotherapy of PDAC patients. To consolidate our findings, the key genes were input into the Oncomine, UALCAN, and LinkedOmics databases to determine relative clinic pathological features and genes. It turned out that CXCL5 participated in an immune response-regulating signaling pathway. The early screening of pancreatic cancer is benefit from longitudinal surveillance programs, and appropriate biomarker such as mucins and imaging-base modalities (4). CXCL5 is significantly higher in pancreatic cancer tissues than normal tissues according to tumor grade and disease stages, it could be used as a biomarker for early diagnosis of PDAC.

The gene CXCL5 is a member of the $\mathrm{C}-\mathrm{X}-\mathrm{C}$ chemokine family, which is believed to be an essential attractant of granulocytic immune cells via targeting to its receptor C-X-C Chemokine Receptor Type 2 (CXCR2) (5). The role of $C X C R 2$ is important for the recruitment of tumor associated neutrophils (TANs) as well as CXCL8, CXCL6 and CXCR1 (6), which suppress antitumor immunity responses in turn. An in vivo study revealed an abundance of tumorinfiltrating T cells, consisting mostly of activated, effector $\mathrm{CD}^{+} \mathrm{T}$ cells in $C X C R 2^{-/-}$hosts (7). It is possible that CXCL5 promotes neutrophil migration through interaction with $C X C R 2$, which then participates in shaping the tumor immune environment (8). In gastric, hepatocellular, and prostate cancers, the interaction between CXCL5 and CXCR2 would dominate cross-talk between cancer cells and macrophages or neutrophils. This kind of cross-talk could promote tumor metastases $(9,10)$. Literature has indicated that CXCL5 can mediate neutrophils to the evasion of immune surveillance by tumor cells via the inhibition of $\mathrm{T}$ cell proliferation and cytokine secretion in laryngeal squamous cell carcinoma (11). In melanoma, the tumor secreted CXCL5 to recruit large quantities of neutrophils and significantly increase lymph node metastases (12). In pancreatic cancer, CXCL5 promoted pancreatic tumor growth by activating several signaling pathways, including protein kinase B (Akt), extracellular signal-regulated kinase (ERK), nuclear factor kappa-light-chain-enhancer of activated B cells (NF-kB) activation, and mutant Kirsten rat sarcoma viral oncogene homolog (Kras) expression (13). Tumors of PDAC patients with higher CXCL5 expression had more intratumoral M2 polarized macrophages, neutrophils, and $\mathrm{IgG}^{+}$plasma cells than patients with lower CXCL5 expression (14). At the same time, necroptotic cells also released CXCL5 to promote cancer cell migration and 
A
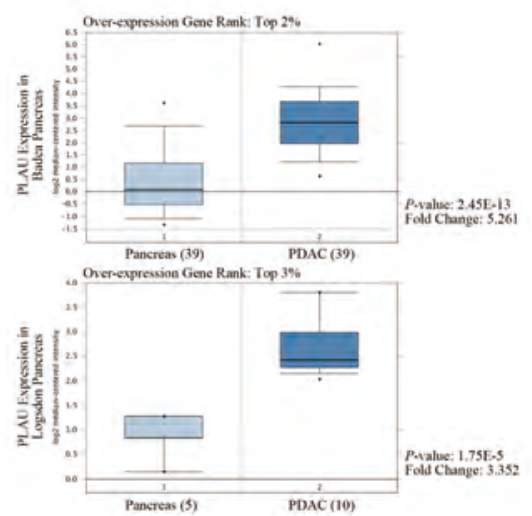

B

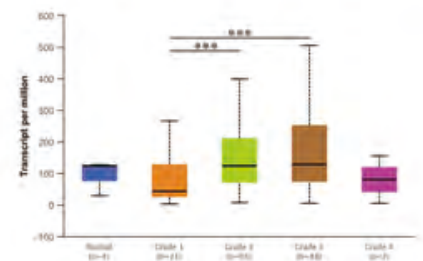

E

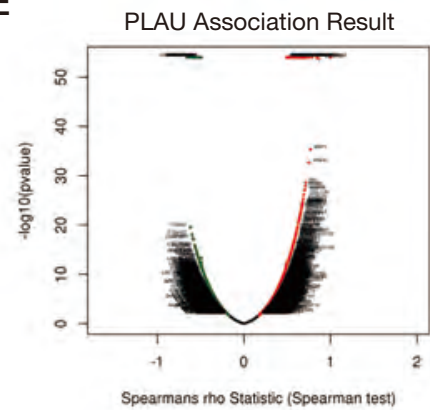

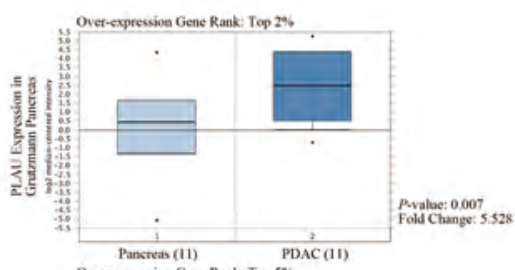

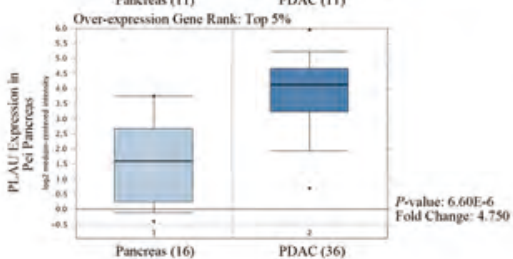

C

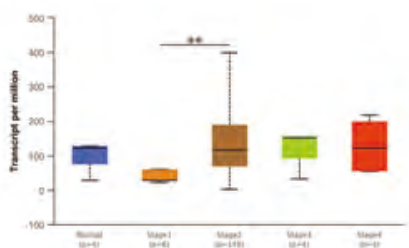

F Negatively Correlated Significant Genes

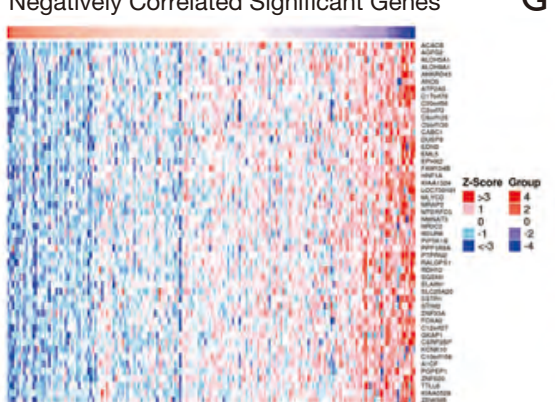

G
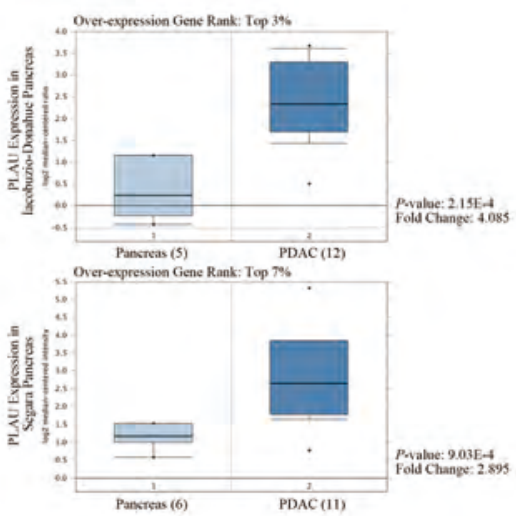

D

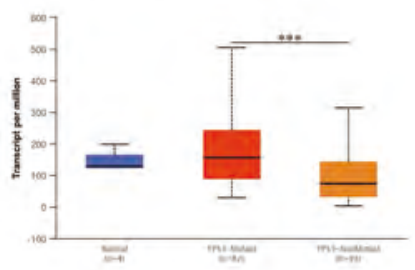

Positively Correlated Significant Genes

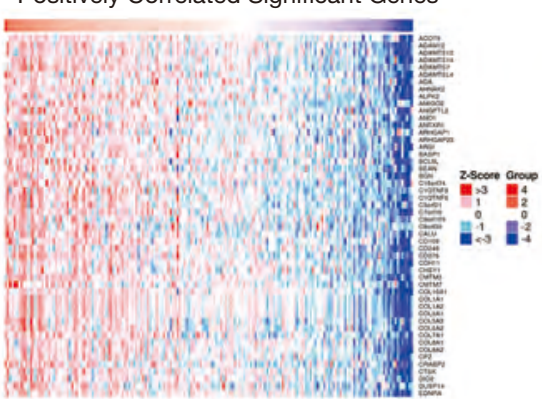

Biological process

Molecule function

KEGG pathway

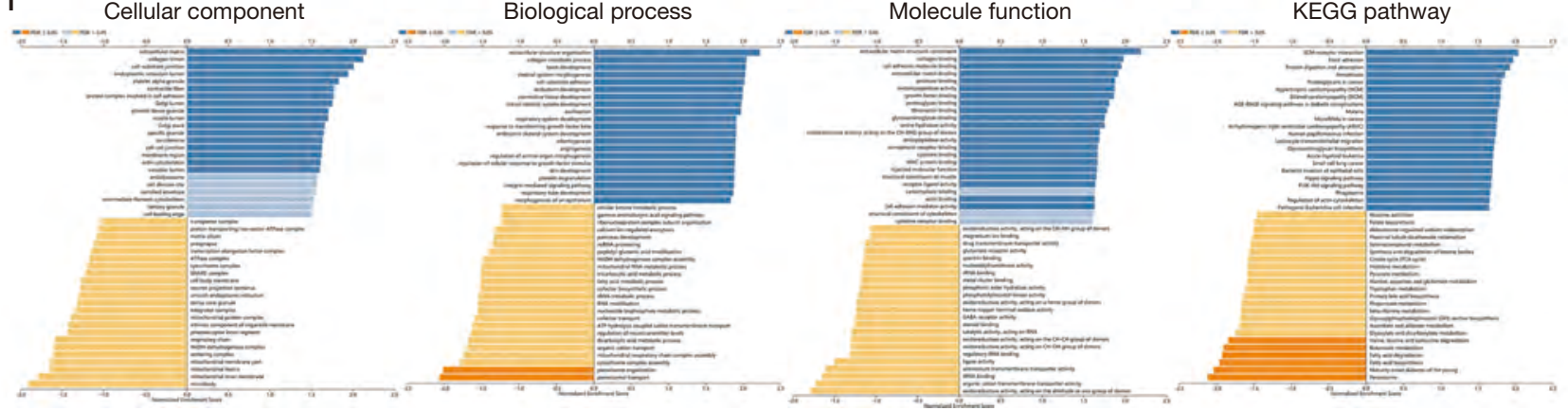

Figure 4 PLAU expression and co-expression genes correlated with PLAU in PDAC. (A) Boxplot showing PLAU mRNA levels in the Badea Pancreas, Grutzmann Pancreas, Iacobuzio-Donahue, Logsdon Pancreas, Pei Pancreas and Segara Pancreas study datasets, respectively; (B) Boxplot exhibiting different transcription levels of PLAU in normal individuals or PDAC patients with grade 1, 2, 3, or 4 tumors; (C) Boxplot exhibiting different transcription levels of PLAU in normal individuals or in PDAC patients in stages 1, 2, 3, or 4; (D) Boxplot exhibiting different transcription levels of PLAU in normal individuals or in PDAC patients with TP53-mutant or TP53-nonmutant; (E) Spearman correlation test was applied to verify correlations between $P L A U$ and DEGs in PDAC. Red represents positively correlated genes and green represents negatively correlated genes; (F,G) heat maps displaying the top 50 negatively and positively related genes of $P L A U$ in PDAC; $(\mathrm{H})$ cellular component, biological process, molecular function, and KEGG pathway analysis of PLAU co-expression genes in PDAC. The blue column indicates the Leading Edge Num, and the orange indicates the FDR. The FDR from GSEA in the figure is 0 . Data are mean \pm SE. *, $\mathrm{P}<0.05 ;{ }^{* *}, \mathrm{P}<0.01$; ${ }^{* * *}, \mathrm{P}<0.001$. FDR, false discovery rate; GSEA, gene set enrichment analysis; KEGG, Kyoto Encyclopedia of Genes and Genomes; DEGs, differentially expressed genes; PDAC, pancreatic ductal adenocarcinoma. 
invasion in pancreatic cancer cells (15). The combined effect of CXCL5 and the receptor CXCR2-ligand helped establish an immunosuppressive microenvironment in PDAC, which has been reported to be associated with unfavorable survival of PDAC patients $(7,16)$. In addition to FOLFIRINOX, CXCL5 could be significantly downregulated by amlodipine in gingival fibroblast cells and upregulated by lipopolysaccharide in astrocytes $(17,18)$. Novel markers such as KRAS mutations, NTRK1-3 fusions and BRCA1/2 mutation had shown targeting therapy potential in PDAC patients. Corresponding drugs including AMG510, entrectinib, larotrectinib and olaparib were still restricted of narrow application and low efficiency (1). CXCL5 inhibitor could be a new choice for precise therapy, and further study of CXCL5 would pave the way to combine FOLFIRINOX and immunotherapy.

The other key gene, PLAU, facilitates digestion of the basal membrane and ECM components. It has been reported that PLAU transcription levels related to matrix metalloproteinases, and played a critical role in tumor proliferation, invasion, and metastasis (19). It has been believed for a long time that $P L A U$ is involved in pancreatic cancer metastasis and invasion, and it was suggested as a prognostic marker in pancreatic cancer patients, associated with poor survival $(20,21)$. Overexpression of PLAU had some effects in the generation of cancer stem cells (22). Silencing of PLAU has been shown to cause a significant reduction in cell invasion and migration of highly metastatic PC-1.0 cells (23).

\section{Conclusions}

The main obstacle to effective PDAC immunotherapy is constituted by its characterization of an immunosuppressive microenvironment. In this study, we found that FOLFIRINOX regulated tumor immunity by reducing expression of the immunosuppressive gene CXCL5. Both the overexpression of CXCL5 and high expression levels in PDAC tissues were associated with a poor prognosis. Our findings may lay a theoretical foundation for the combined therapy of FOLFIRINOX and immunotherapy, which might offer increased survival benefits to patients with PDAC.

\section{Acknowledgments}

Funding: This work was supported by the National Natural Science Foundation of China $(81602091,81702048)$.

\section{Footnote}

Reporting Checklist: The authors have completed the MDAR reporting checklist. Available at http://dx.doi.org/10.21037/ gs-20-828

Conflicts of Interest: All authors have completed the ICMJE uniform disclosure form (available at http://dx.doi. org/10.21037/gs-20-828). The authors have no conflicts of interest to declare.

Ethical Statement: The authors are accountable for all aspects of the work in ensuring that questions related to the accuracy or integrity of any part of the work are appropriately investigated and resolved. The study was conducted in accordance with the Declaration of Helsinki (as revised in 2013).

Open Access Statement: This is an Open Access article distributed in accordance with the Creative Commons Attribution-NonCommercial-NoDerivs 4.0 International License (CC BY-NC-ND 4.0), which permits the noncommercial replication and distribution of the article with the strict proviso that no changes or edits are made and the original work is properly cited (including links to both the formal publication through the relevant DOI and the license). See: https://creativecommons.org/licenses/by-nc-nd/4.0/.

\section{References}

1. Klaiber U, Hackert T, Neoptolemos JP. Adjuvant treatment for pancreatic cancer. Transl Gastroenterol Hepatol 2019;4:27.

2. Ychou M, Conroy T, Seitz JF, et al. An open phase I study assessing the feasibility of the triple combination: oxaliplatin plus irinotecan plus leucovorin/ 5 -fluorouracil every 2 weeks in patients with advanced solid tumors. Ann Oncol 2003;14:481-9.

3. Conroy T, Hammel P, Hebbar M, et al. FOLFIRINOX or gemcitabine as adjuvant therapy for pancreatic cancer. $\mathrm{N}$ Engl J Med 2018;379:2395-406.

4. Singhi AD, Koay EJ, Chari ST, et al. Early detection of pancreatic cancer: opportunities and challenges. Gastroenterology 2019;156:2024-40.

5. Dang $\mathrm{H}, \mathrm{Wu} \mathrm{W}$, Wang B, et al. CXCL5 Plays a Promoting Role in Osteosarcoma Cell Migration and Invasion in Autocrine- and Paracrine-Dependent Manners. Oncol Res 2017;25:177-86. 
6. Bonavita O, Massara M, Bonecchi R, et al. Chemokine regulation of neutrophil function in tumors. Cytokine Growth Factor Rev 2016;30:81-6.

7. Chao T, Furth EE, Vonderheide RH. CXCR2-Dependent Accumulation of Tumor-Associated Neutrophils Regulates T-cell Immunity in Pancreatic Ductal Adenocarcinoma. Cancer Immunol Res 2016;4:968-82.

8. Zhou SL, Dai Z, Zhou ZJ, et al. CXCL5 contributes to tumor metastasis and recurrence of intrahepatic cholangiocarcinoma by recruiting infiltrative intratumoral neutrophils. Carcinogenesis 2014;35:597-605.

9. Roca H, Jones JD, Purica MC, et al. Apoptosis-induced CXCL5 accelerates inflammation and growth of prostate tumor metastases in bone. J Clin Invest 2018;128:248-66.

10. Zhou Z, Xia G, Xiang Z, et al. A C-X-C chemokine receptor type 2-dominated cross-talk between tumor cells and macrophages drives gastric cancer metastasis. Clin Cancer Res 2019;25:3317-28.

11. Zhang D, Zhou J, Tang D, et al. Neutrophil infiltration mediated by CXCL 5 accumulation in the laryngeal squamous cell carcinoma microenvironment: A mechanism by which tumour cells escape immune surveillance. Clin Immunol 2017;175:34-40.

12. Soler-Cardona A, Forsthuber A, Lipp K, et al. CXCL5 facilitates melanoma cell-neutrophil interaction and lymph node metastasis. J Invest Dermatol. 2018;138:1627-35.

13. Li A, King J, Moro A, et al. Overexpression of CXCL5 is associated with poor survival in patients with pancreatic cancer. Am J Pathol 2011;178:1340-9.

14. Zhang R, Liu Q, Peng J, et al. CXCL5 overexpression predicts a poor prognosis in pancreatic ductal adenocarcinoma and is correlated with immune cell infiltration. J Cancer 2020;11:2371-81.

15. Ando Y, Ohuchida K, Otsubo Y, et al. Necroptosis in pancreatic cancer promotes cancer cell migration

Cite this article as: $\mathrm{Wu} M Y$, Shen $\mathrm{M}, \mathrm{Xu} M D, \mathrm{Yu} Z Y$, Tao M. FOLFIRINOX regulated tumor immune microenvironment to extend the survival of patients with resectable pancreatic ductal adenocarcinoma. Gland Surg 2020;9(6):2125-2135. doi: $10.21037 /$ gs-20-828 and invasion by release of CXCL5. PLoS One 2020;15:e0228015.

16. Rozengurt E, Sinnett-Smith J, Eibl G. Yes-associated protein (YAP) in pancreatic cancer: at the epicenter of a targetable signaling network associated with patient survival. Signal Transduct Target Ther 2018;3:11.

17. Lauritano D, Martinelli M, Baj A, et al. Drug-induced gingival hyperplasia: An in vitro study using amlodipine and human gingival fibroblasts. Int J Immunopathol Pharmacol 2019;33:2058738419827746.

18. O'Sullivan SA, O'Sullivan C, Healy LM, et al. Sphingosine 1-phosphate receptors regulate TLR4-induced CXCL5 release from astrocytes and microglia. J Neurochem 2018;144:736-47.

19. Choong PF, Nadesapillai AP. Urokinase plasminogen activator system: a multifunctional role in tumor progression and metastasis. Clin Orthop Relat Res 2003;(415 Suppl):S46-58.

20. Cantero D, Friess H, Deflorin J, et al. Enhanced expression of urokinase plasminogen activator and its receptor in pancreatic carcinoma. Br J Cancer 1997;75:388-95.

21. Wang W, Abbruzzese JL, Evans DB, et al. Overexpression of urokinase-type plasminogen activator in pancreatic adenocarcinoma is regulated by constitutively activated RelA. Oncogene 1999;18:4554-63.

22. Asuthkar S, Stepanova V, Lebedeva T, et al. Multifunctional roles of urokinase plasminogen activator (uPA) in cancer stemness and chemoresistance of pancreatic cancer. Mol Biol Cell 2013;24:2620-32.

23. Liu P, Weng Y, Sui Z, et al. Quantitative secretomic analysis of pancreatic cancer cells in serum-containing conditioned medium. Sci Rep 2016;6:37606.

(English Language Editor: J. Jones) 

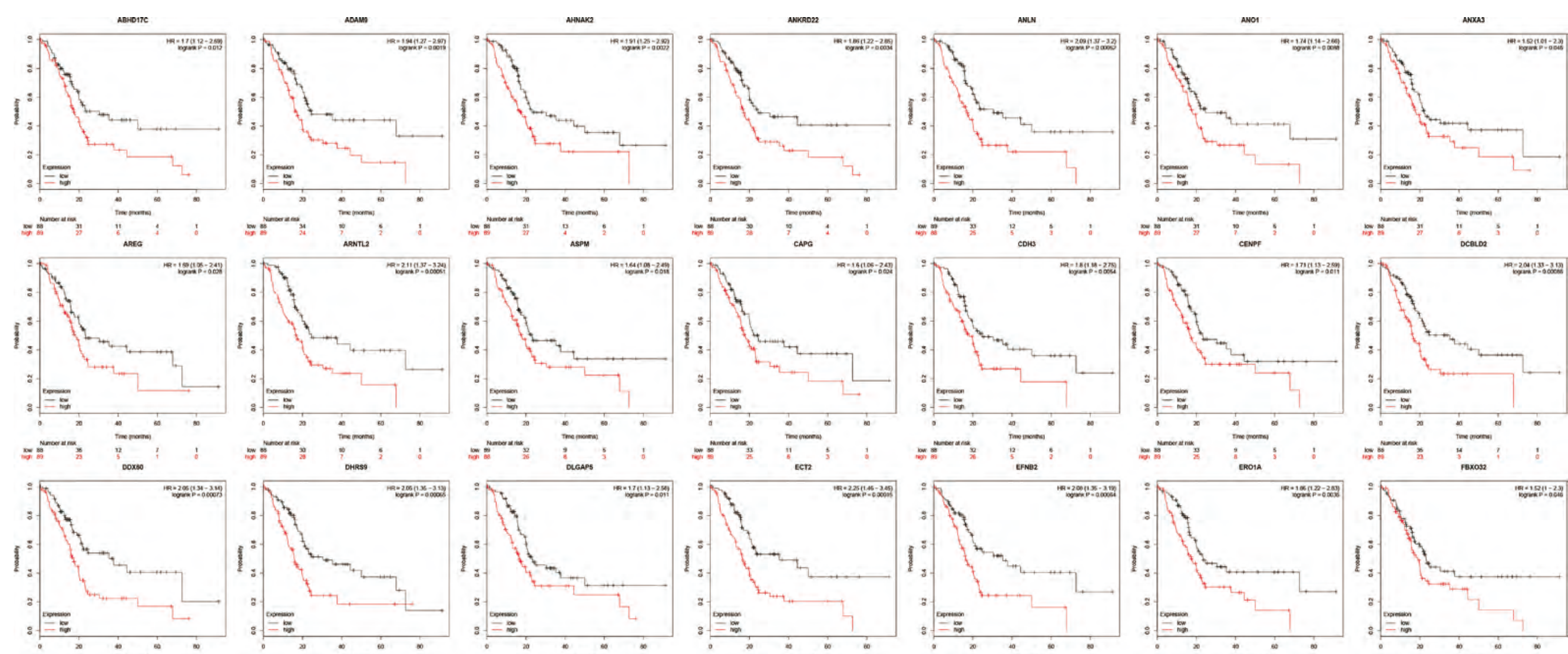

ᄂ ᄀ
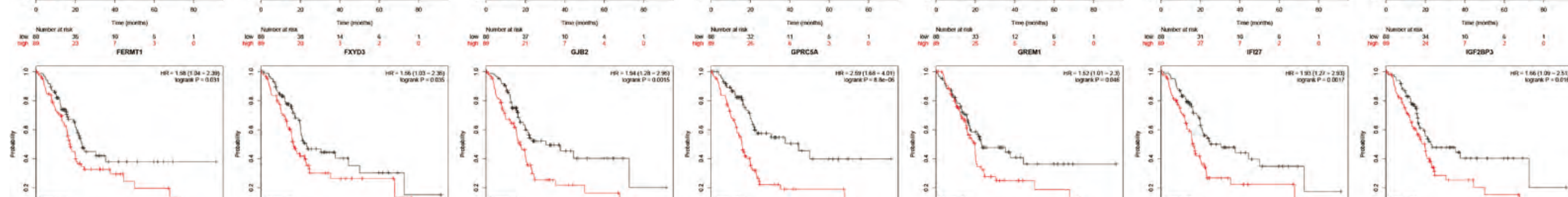

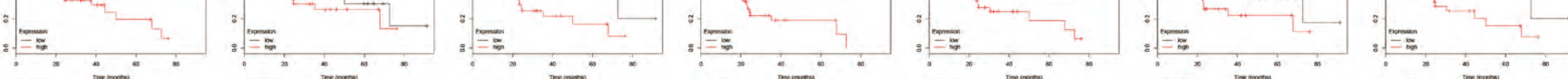
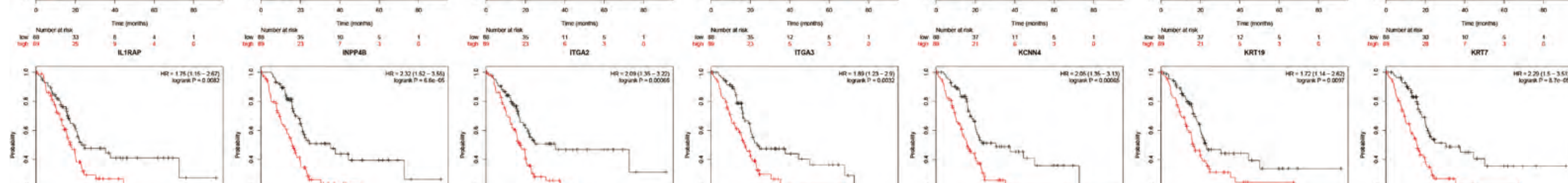

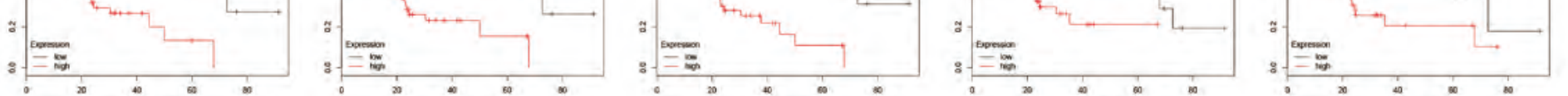

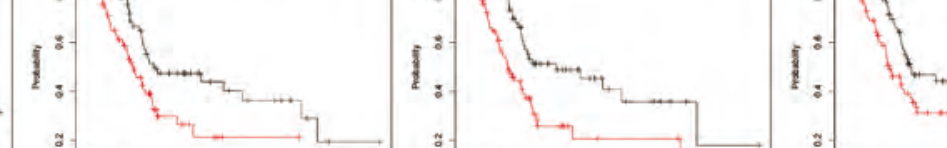

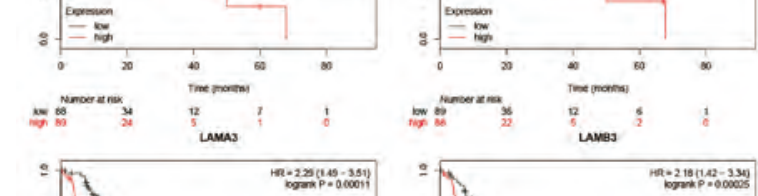

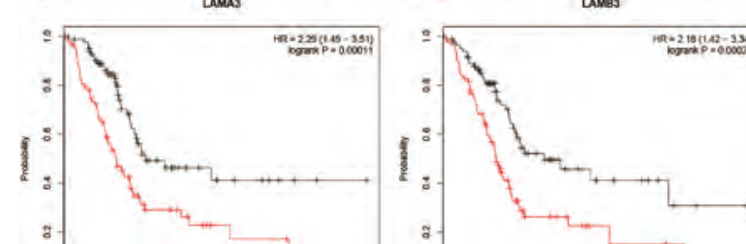

:

$=0$

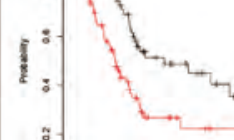

$=$

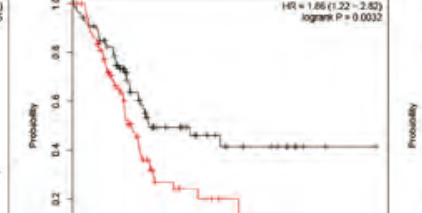

$1_{:-1}^{:-1+4,4}$

Fil

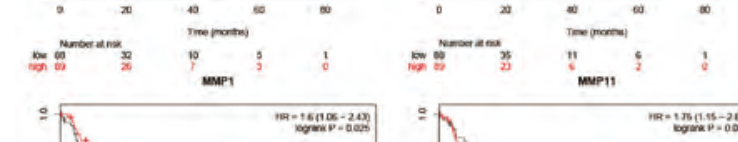

:

$=$

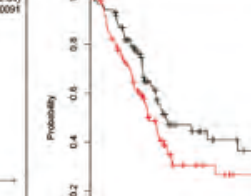

: $:$ :

$1:-2$
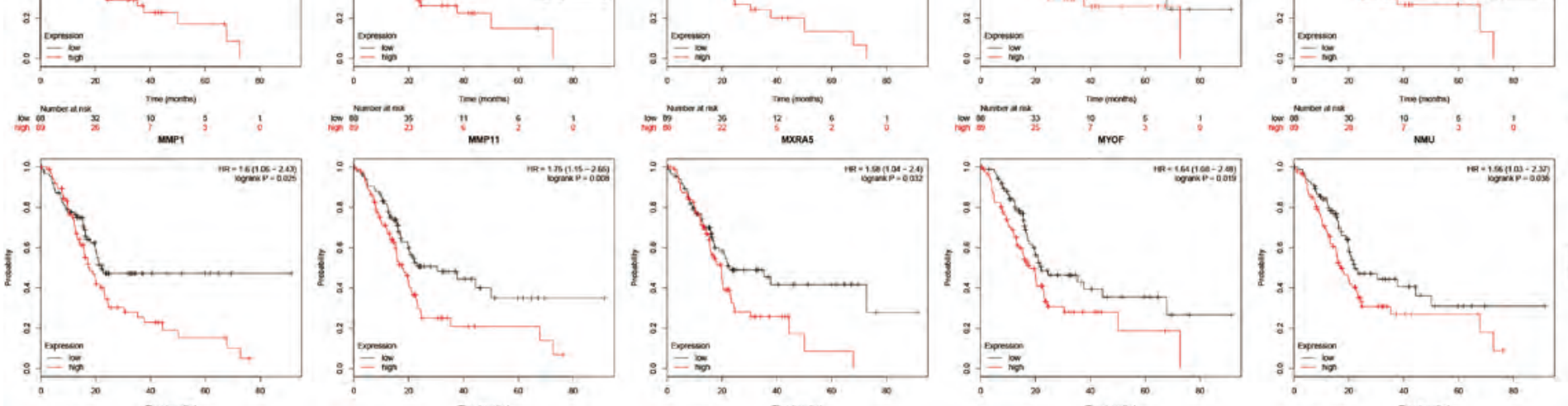

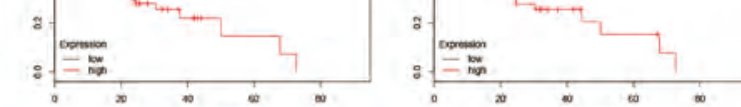

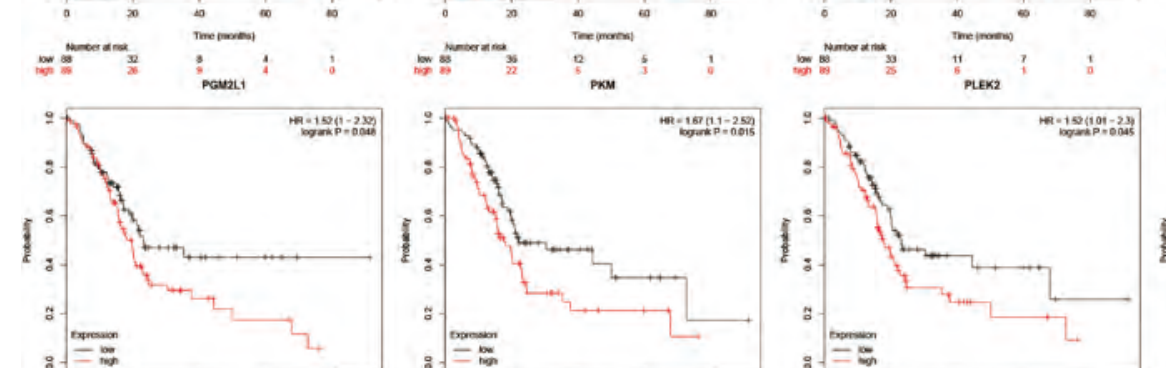

=

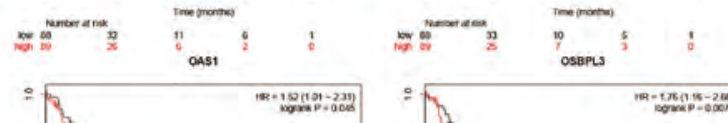

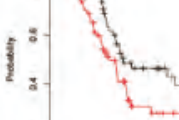

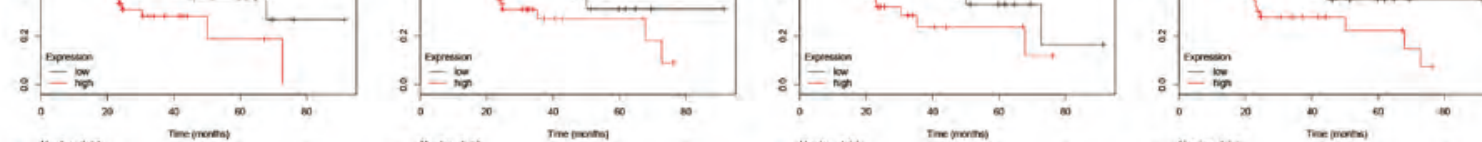

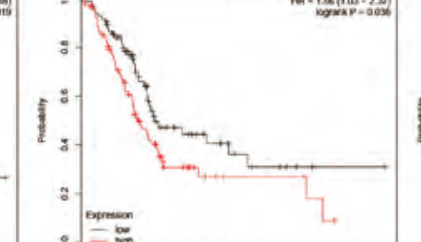

$1:$ :

1: $:$ :

F

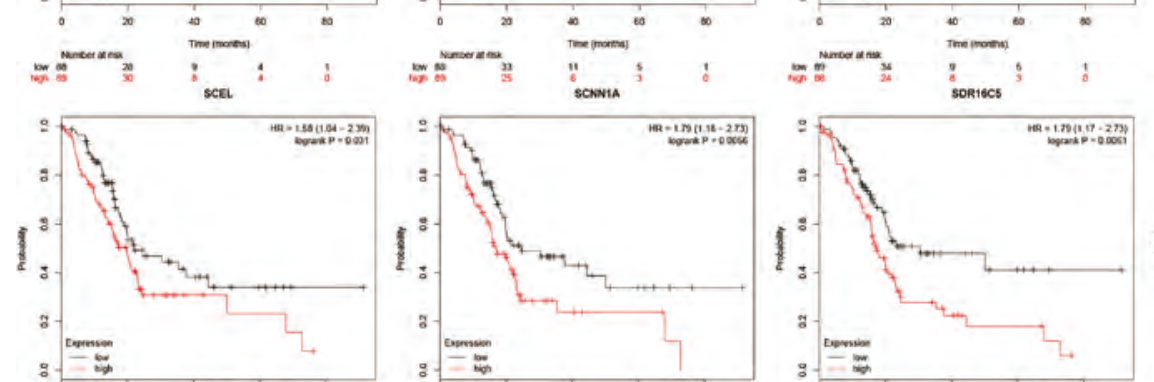

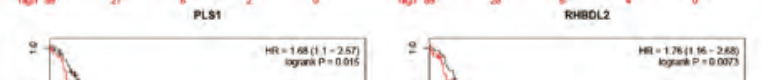

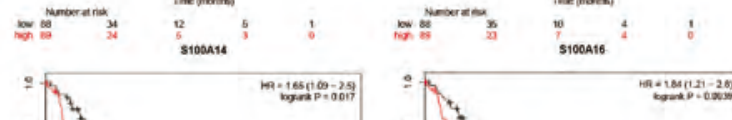

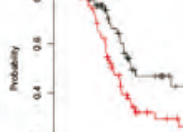

: $: 2$

$1:$ :

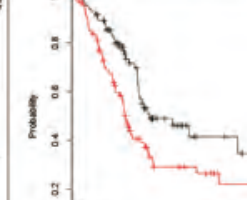

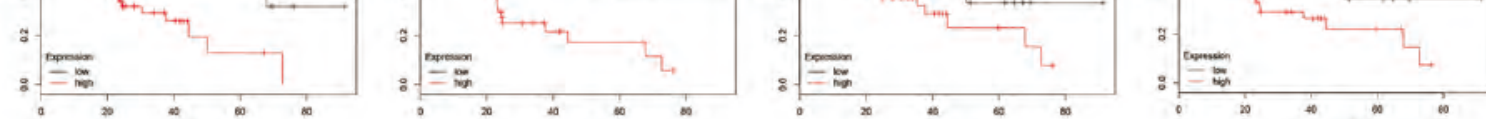

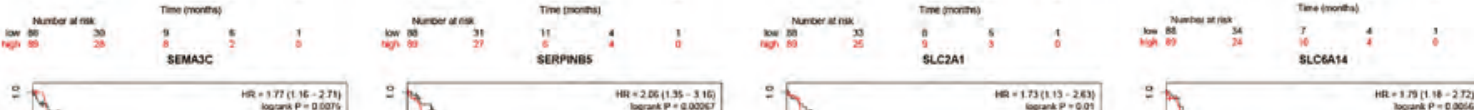

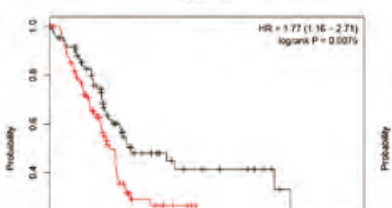

$1:-2$

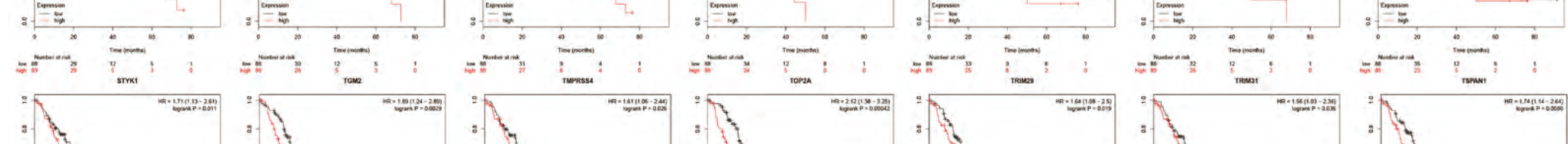

$=4=4$

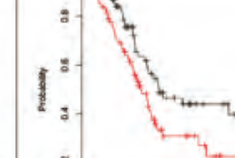

:

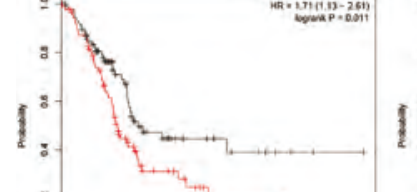

$1:-1,-4$

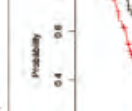

1:-

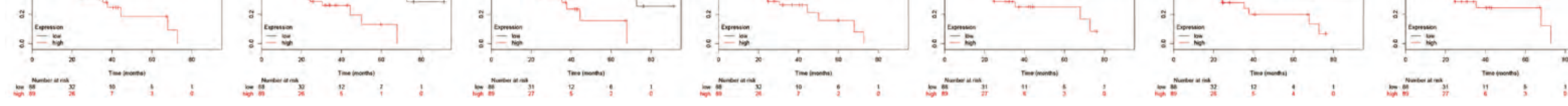

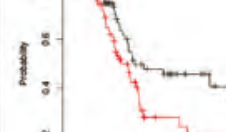

$1:-2$.

Figure S1 Prognostic values of overlapping DEGs in the GSE16515, GSE15471, and GSE28735 data sets. DEGs, differentially expressed genes. 